\title{
Aprendizaje de la Lecto-Escritura Inicial como Factor Pronóstico del Rendimiento Escolar Ulterior $\left.{ }^{*}\right\}$
}

\author{
Dr. Alejandro Manterola A.' ; Sr. Pultricio Avendaño P. ${ }^{2}$; Sr. Jaime Cotronco E. ${ }^{2}$; \\ Dr. Alfiedo Avendaño B. ${ }^{3}$; Dr. Carlos Valenz.uela $Y^{4}$ \\ Early Ability to Read and to Whrite as Index \\ of Late A cademic Performance
}

\begin{abstract}
One hundred mineteen mentally normal children, from middle low and low socioecononic strata. living in the urtan northern area of Santiago. Were evaluated in 1975 for reading and writting cornpetence, during the second term of the third grade elementary schooling. A developmental multitaced follow-up was providea for them since their elementary school entrance in $\mathbf{1 9 7 3}$ up to now. Besides, in 1982 they were examined through a test bartcry in order to get an evenly appraisal of their acquaintance in reading, writting, solving basic arithmetical tasks and ability to design simple tridimensional objects. Direct, analogous and significant statistical correlations were found between reading and writting performance at the third elementary grade and those parameters selected as index of academic progress seven years after. Most of the children disabled in reading and witting at mean ages of 8.9 yr. shuwed lattes. a rather poor academic performance on the average and no one reached optimal total scores. The lowest scores were got in arithmethic solving abilities and in designing usual models. On the contrary, children that easily learned how to read and write did much beter, even when some of them periormed not so well, requiring individuitized analysis under work-up. These relationship appear to be in some wily independent of the late rcadin! and writting level, since more than half of the subjects ( $53 \%$ were able to manage easily on the matter. (Key words: Academic perfomance. Arithmetic. Early. Prediction. Reading. Writting).
\end{abstract}

En Marzo de 1973 se inició en la Unidad de Crecimiento y Desarrollo del Hospital Infantil Roberto del Río un estudio longitudinal de niños que ingresan al primer Año Básico de las escuelas del Area Norte de Santiago, a una edad promedio de 6. años. Para este objeto se eligió al azar una

1. Médico Neurólogo Departamento de Pediatría, Fa cultad de Medicina, División Ciencias Médicas Norte, Universidad de Chile.

2. Estudiantes Carrera de Medicina, Facultad de Medicina, División Ciencias Médicas Norle, Lnjversidad de Chile.

3. Médico Departamento de Pediatría, Facultad de Medicina, División Ciencias Médicas Norte, Cniversidad de Chile.

4. Médico Departamento Biologia Celular y Genética, Facultad de Medicina, División Ciencias Médicas Norte, Univ. de Chile.

(*) Trabajo financiado por Grant M 797/8555, Univer. sidad de Chile, Dirección Gentral Académica y Estudiantil, Departamento de Investigación y Biblioiecal.

Centro de Crecimiento y Desarrollo, Servicio Salud Metropolatano Norte, Hospital Roberto del Río. mnuestra de 790 sujetos de la cohorte total de 13.270 matriculados en Marzo de ese año en las distintas escuelas del sector. El grupo de estudio se sometió a un programa de control seriado đe carácter multiprofesional, incluyendo médicos, enfermeras: nutricionistas, sicólogos, profesores. asistente social y auxiliares de atención médica, cuyo diseño fue motivo de una publicación ${ }^{1}$.

A partir de 1975 se efectuaron en estos niños diversos estudios de orden biológico, psicológico y psicosocial. Lino de ellos tuvo como objetivo conocer la suficiencia del aprendizaje escolar: tomando como indice la habilidad para adquirir la lecto-escritura, transcurridos 5 semestres completos del programa oficial del Ministerio de Educación, según las normas técnicas vigentes. Los detalles de la metodología, y los resultados obtenidos fueron subsecuentemente publica$\operatorname{dos}^{2-3-4-5}$. Una ponderación estadística con metodología bayesiana de los resultados se realizó posteriormente ${ }^{6}$, un estudio de validación de las relaciones entre presencia de alteraciones neurológicas y bajo rendimiento escolar efectuado en una población diferente de niños del sector norte de Santiago. completó la primera fase del estudio? 
En esta ocasión se da cuenta de ios resultados de la correlación entre la capacidad de aprendizaje inicial en lecto-escritura determinada a nivel del tercer Año Básico y el rendimiento y habilidades escolares adquiridos 7 años mas tarde. Para este efecto, se comparan los niños que tuvieron dificultad inicial con aquellos que aprendieron a leer $y$ a escribir sin ninguna dificultad. E1 objetivo ha sido evaluar el supuesto que la demora inicial del aprendizaje, excluido el retardo mental, es un factor predictivo de rendimiento académico a lo largo de los años.

\section{METODOLOGIA Y CASUISTICA}

Con el objeto de poner a prueba la hipótesis de trabajo, un grupo importante de los niños estudiados en 1975 fueron sometidos a un reestudio programado del rendimiento académico siete años después. En este estudio las edades de los sujetos tuvieron un rango de 15 a 17 años, con promedio 16 años.

El estudio incluye los datos de continuidad $y$ progresión escolar en el curso del tiempo y una estimación objetiva de las habilidades alcanzadas en lecto-escritura, ortografia, caligrafía, dibujo tridimensional y aritmética elemental. Se consideró como un elemento de evaluación general los datos de la secuencia y progresión de la escolarjdad desde el año 1974 a 1982 , con referencia al número o nombre de las escuelas a que asistió en el periodo, señalando si requirió cursos especiales. Se registró también el dato anamnéstico de la asistencia escolar en los últimos 2 afios y se evalú los certificados y las notas obtenidas al finalizar el año escolar en 1981. Las mediciones objetivas se hicieron mediante un conjunto de 15 pruebas totalmente similates para cada niño bajo estudio (Tabla 1). Cada prueba, compuesta de un número variable de items, fue puntuada en escala de 0 - 3, lo que tradujo un puntaje de máxima eficiencia de 45 puntos.

Los casos corresponden al grupo seguido longitudinalmente en quienes fue posible documentar los datos tanto del estudio inicial como en 1982. Así, de los 208 casos estudiados en 1975 se pudieron reexaminar en forma completa 153 niños. Los restantes 55 niños no concurrieron al examen de control ni fue posible hacerlo en el hogar (cambio de domicilio, negativa de los niños a dejarse examinar, etc.). Para los efectos de la correlación entre la capaciđad de aprendizaje de la lecto-escritura inicial y el rendimiento ulterior se consideraron sólo 119 niños que exhibieron un coeficiente intelectual de 80 o más en el estudio realizado en 1975. Se excluyeron los casos con coeficiente menor para eliminar el sesgo que significa el déficit intelectual, aspecto que sera considerado en un estudio separado. Las significaciones estadisticas se calcularon por una prue. ba $Z$ de proporciones o por distribución de Poisson en caso de números pequeños.

Tabla I.

Resumen de la Prueba Global de Conocimientos y Habilidades.

1) Traducción a números de 5 cantidades eseritas (24 la más simple. y 16.314 la más compleja).

2) Colocar palabras ornitidas en una trase (total 4 frases).

3) Laet en voz baja una pequeña historia escrita y contestar luego preguntas de comprensión del tema. (Si ei niño no contestaba se le léa la historieta en voz alta).

4) 3 sumas de cifras de 3 y 4 dígitos.

5) 3 restas de 3 y 4 dígitos.

6) Multiplicación de cifras de 2 digitos por una cifra, 3 dígitos por 2 cifras y 4 dígitos por 3 cifras.

7) División de 2 dígitos por una cifra, 3 dígitos por 2 cifras y 4 dígitos por 2 cifras (guarismos divisibles, sin decimales).

B) Dibujo de memoria de una botella con etiqueta, una silla con sus cuatro patas $y$ un dado visto por 3 caras.

9) Dictado de tres frases elaboradas.

10) Dictado de una frase de 22 palabras.

11) Evaluación de la caligrafía empleada para escribir la frase en un papel sin líneas.

12) Estimación de las fallas de ortografía cometidas en el escrito de la frase dictada (Prueba 10).

13) Dadas tres secuencias de fracciones se le pide al sujeto que las continúe; por ejemplo: $1 / 10,1 / 20,1 / 30 \ldots$ etc.

14) Llenar espacios en blanco en 3 progresiones o regresiones; por ejemplo: $1.2,1.0, \ldots ., 0.6, \ldots . ., 0.2$.

15) Determinar 6 equivalencias de fracciones a números enteros con decimales, preguntando sí, por ejempk, $1 / 4$ es igual o no a la mitad de 0,5 . 


\section{RESULTADOS}

El estudio comparativo de la capacidad de aprendizaje de la lecto-escritura a nivel del tercer Año Básico con el rendjmiento escolar ulterior se desarrolla en las tablas a continuación.

En la Tabla 2 se dan las cifras obtenidas al correlacionar el aprendizaje escolar inicial con el progreso general estimado en función de la progresión anual y de las notas obtenidas al termino del año precedente (1981). Se estimó el progreso como óptimo si no hubo repetición de cursos y las notas de promoción fueron superiores a 5 (escala de 1 a 7). La calificación de regular corresponde a sujetos que repitieron hasta un curso, obteniendo a fines de 1981 una o más notas entre 4 y 5 o ambas. Se calificó como malo el progreso escolar irregular, con dos o más repeticiones de año, una o más notás inferiores a 40 ambas. Fueron excluidos de este análisis dos niños que en algún momento tuvieron que ser retirados transitoriamente del sistema educacional por razones extraacadémicas (problemas económico-sociales) pero que, salvado el momento critico, se reincorporaron al sistema escotar, por to cual el número de casos incluidos en Tabla 2 es de 117. En ella se muestra que los sujetos sin dificultades de aprendizaje inicial fueron claramente mejor calificados en su progreso que quienes aprendieron a leer con lentitud. El análisis estadístico demuestra que el número de sujetos mal calificados que habian tenido dificultad inicial en lecto -..escritura era significativamente más elevado $(Z=3.536 p<0.0003)$ respecto a los que aprendieron a leer sin demora. Es también llamativo que no hubo caso alguno con dificultad inicial de lecto-escritura que lograra una calificación satisfactoria siete años más tarde. La diferencia con-los sujetos bien calificados en ambos aspectos es también significante (Poisson $<0.002$ )

En la Tabla 2 también se relaciona la capacidad de aprendizaje inicial de la lecto-escritura con los resultados de la prueba global de habilidades adquiridas, luego de 10 años de programa escolar. Los puntajes obtenidos en esta prueba se ordenaron en 3 franjas. Dentro de la escala alta se ubicaron 16 que obtuvieron entre 40 y 45 puntos (13.5\% de los 119 nifos estudiados); en la zona intermedia quedaron los 80 que obtuvieron entre 30 y 39 puntos $(67 \%)$; y en ta baja, $\operatorname{los} 28$ sujetos que obtuvieron menos de 30 puntos (19.5\%). En esta particular correlación se muestra que sólo entre los sujetos que aprendieron a leer rápidamente estaban aquellos que fueron capaces de obtener los más altos puntajes en la prueba objetiva utilizada. Por el contrario, de los niños con buen aprendizaje inicial sólo hay un número relativamente bajo $(10 \%)$ que obtuvieron pobres resultados, mientras que un bajo puntaje fue obtenido por más de la mitad (55\%) de los sujetos que presentaron franca dificultad inicial de aprendizaje. Las diferencias de estas cifras desde el punto de vista estadístico son altamente significativas $(Z=3.74 p<0.002)$. Hubo tam. bién diferencias entre el número de sujetos con bajo rendimiento que aprendieron a leer sin dificultad con los que lo hicieron con retrasos moderados $(Z=1.66 \mathrm{p}<0.0502)$ y entre estos últimos y los que aprendieron con muchas dificultades $(Z=1.24 \mathrm{p}>0.1000)$. Los niños que habiendo aprendido a leet con facilidad tuvieron un desempeño pobre en las pruebas objetivas de evaluación, serản motivo de un análisis especial.

Tabk 2.

Lecto-Fscritura Inicial en Relación al Progreso $y$ al Nivel de Conocimientos Evaluados 7 Años Después.

\begin{tabular}{|c|c|c|c|c|}
\hline \multirow[b]{2}{*}{ Readimenlal } & \multicolumn{2}{|c|}{$\begin{array}{l}\text { Diticultad Inicial' } \\
\text { dt Lecto-Fscritura }\end{array}$} & \multicolumn{2}{|c|}{ Giadu de Dificultad } \\
\hline & $\mathrm{N}_{0}$ & $\mathrm{Si}_{\mathrm{i}}$ & Hoderads & Mafcado \\
\hline $\begin{array}{l}\text { A. Progreso } \\
\text { Atidémisu * }\end{array}$ & $N=8 R$ & $N=29$ & $N=16$ & $N=13$ \\
\hline $\begin{array}{l}\text { - Optimo } \\
\text { - RepulaI } \\
\text { - Bsice }\end{array}$ & $\begin{array}{r}26(29 \%) \\
53(61 \%) \\
9110 \%\end{array}$ & $\begin{array}{c}0 \\
16 ! 56 \%) \\
13(44 \%)\end{array}$ & $\begin{array}{l}0 \\
9 \\
7\end{array}$ & $\begin{array}{l}0 \\
? \\
b\end{array}$ \\
\hline $\begin{array}{l}\text { B. Punehas } \\
\text { Converum atos: }\end{array}$ & $N=89$ & $\lambda=30$ & $N=17$ & $N=1.3$ \\
\hline $\begin{array}{l}\text { - Alto } \\
40-45 p(w)\end{array}$ & $16(17 \%)$ & 0 & 0 & $\vdots$ \\
\hline $\begin{array}{l}\text { Mcdio } \\
\text { (30.39 p(4s) }\end{array}$ & $64(83 \%)$ & $16(53 \%)$ & 10 & 5 \\
\hline $\begin{array}{l}- \text { Bujo } \\
1<30 \text { ptos })\end{array}$ & $9110 \%$ & $14(47 \%)$ & 7 & $?$ \\
\hline
\end{tabular}

. Se excluyen 2 casos con propesow interrumpido por tausas ccoric mico-saciales.

Al comparar separadamente el resultado de las pruebas para medir la calidad y rapidez de la lecto-escritura en la bateria aplicada en el último corte con el grado de dificultad inicial de estas habilidades (Tabla 3) se aprecia que la gran mayoría $(91 \%)$ de los niños sin dificultad inicia obtuvo puntajes altos en las pruebas de lectoes. critura ulterior y que sólo 1 de los 89 riños de esta categoría cometía errores importantes. En cambio, de los 30 niños con dificultades iniciales en lecto-escritura 11 (36\%) seguían cometiendo algunos errores y $3(10 \%)$ todavia leian defec. tuosamente. Estas cifras, en el anälisis estadístico, traducen diferencias altamente significativas $(Z=3.92 \mathrm{p}<0.00008$ ) entre ambos grupos.

Cuando se relaciona el rendimiento en las habilidades aritméticas, sumando el puntaje obtenido en las pruebas $4,5,6,7,13,14,15$ de la bateria con el aprendizaje inicial de la lecto-lectu- 
ra, los resultados obtenidos al cruzar ambas variables permiten señalar que en los niños sin ninguna dificultad de aprendizaje inicial el rendimiento en aritmética tendió a ser bueno o al menos aceptable. Por el contrario, en el grupo de nixos con marcada dificultad de aprendizaje inicial no hubo ningún caso que tuviera calificaciones optimas y la mayoria tuvo un mal resultado, diferencia que es estadisticamente significativa (rendimiento deficiente comparado en ambos grupos, $Z=3.11 \mathrm{p}<0.005$ ). Si se considera sólo las operaciones aritméticas elementales (suma. resta, multiplicación y división) las diferencias son tambiein apreciables entre ambos grupos $(Z=3.95 p<0.00008)$.

\section{Tabla 3.}

Lecto-Escritura Inicial en Relación a Habjlidades en Lectura y Aritmética Básica Adquiridas ? Años más Tarde.

\begin{tabular}{|c|c|c|c|c|}
\hline \multirow[b]{2}{*}{$\begin{array}{l}\text { Puntaje en Batetia } \\
\text { de: Habilidales }\end{array}$} & \multicolumn{2}{|c|}{ Duscultad Inscinl } & \multicolumn{2}{|c|}{ Gratso do ldilicuttud } \\
\hline & $\begin{array}{l}\text { No } \\
N=89\end{array}$ & $\underset{v=30}{S i}$ & $\begin{array}{l}\text { Blodicadal } \\
N=l\end{array}$ & $\begin{array}{c}\text { Madeitdit } \\
N=1 ?\end{array}$ \\
\hline \multicolumn{5}{|l|}{ A. Lesto-liseritura: } \\
\hline alto $(12-15 p(a s)$ & $91<90$ & $16(5390)$ & 5 & 7 \\
\hline medio ildg-11 ptosj & $3(0 \times \infty)$ & $1](36 \%)$ & 6 & 5 \\
\hline baju $(<d i q$ ptos) & $101 \%$ & 03 (1) iond & 2 & 1 \\
\hline \multicolumn{5}{|l|}{ H. Nrilincticin: } \\
\hline alo $\{1 \mathrm{~B}-21$ ptos & $31) 134 \%$ & i) & 3 & i \\
\hline $\begin{array}{l}\text { medio }(12-17 \text { peses }) \\
\text { bajo }(<12 \mathrm{ptos} !\end{array}$ & $52658 \%$ & $19163^{\circ}$ & $\begin{array}{r}13 \\
4\end{array}$ & $\stackrel{1}{1}$ \\
\hline balo $(<1$ & & $111377^{4040}$ & & \\
\hline
\end{tabular}

La capacidad de hacer dibujos tridimensionales (Prueba 8) fue tambien de mejor calidad en los niños sin dificultades iniciales de lecto-escritura que en los niños que presentaron dificultades (Tabla 4 y Figuras 1 A y B. Los aspectos que se consideraron más relevantes en la calificación fueron la perspectiva, la proyección tridimensional. la seguridad de] trazo y los elementos artisticos introducidos (sombreado,perfiles, armonía de los segmentos, etc.). Aún cuando un número importante $(31 \%)$ de los niños sin dificultad de aprendizaje inicial hicieron un dibujo tridimensional deficiente, esta proporción es doblada en los escolares que aprendieron a leer con tardanza, diferencia que es estadisticamente significativa $(Z=2,795 \mathrm{p}<0.0031)$. Por contraste solamente 1 de los 30 sujetos que aprendieron a leer dificultosamente realizaron dibujos caralogados en general como "bueno", mientras que la mayoria se consideró "deficiente". La proporción de dibujos de calidad "aceptable" fue también mayor en los sujetos sin dificultades del aprendizaje inicial. Las diferencias estadísticas de estas cifras en ambos grupos son significativas $(Z=1.793 p<0.0379)$.
Tabla 4 .

Lecto-Escritura Inicjal en Relación a Habilidad en Dibujo Tridimensional

\begin{tabular}{|c|c|c|c|c|}
\hline \multirow[b]{2}{*}{ Culidat del Dibuio } & \multicolumn{2}{|c|}{ 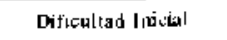 } & \multicolumn{2}{|c|}{ Grade de Dificuliad } \\
\hline & $\begin{array}{c}\text { No } \\
n=84\end{array}$ & $\begin{array}{c}S i \\
N=39\end{array}$ & $\begin{array}{c}\text { Moderado } \\
x=1 ?\end{array}$ & $\begin{array}{c}\text { Marcado } \\
N=13\end{array}$ \\
\hline $\begin{array}{l}\text { Optıma } \\
\text { Accprabls: } \\
\text { Deticiente }\end{array}$ & $\begin{array}{l}12113 \%) \\
49(55 \%) \\
28(31 \%)\end{array}$ & $\begin{array}{l}1(0,3 \%) \\
11,36 \% \\
18,60 \%\end{array}$ & $\begin{array}{c}0 \\
\vdots \\
10 \\
10\end{array}$ & $\begin{array}{l}1 \\
4 \\
k\end{array}$ \\
\hline
\end{tabular}
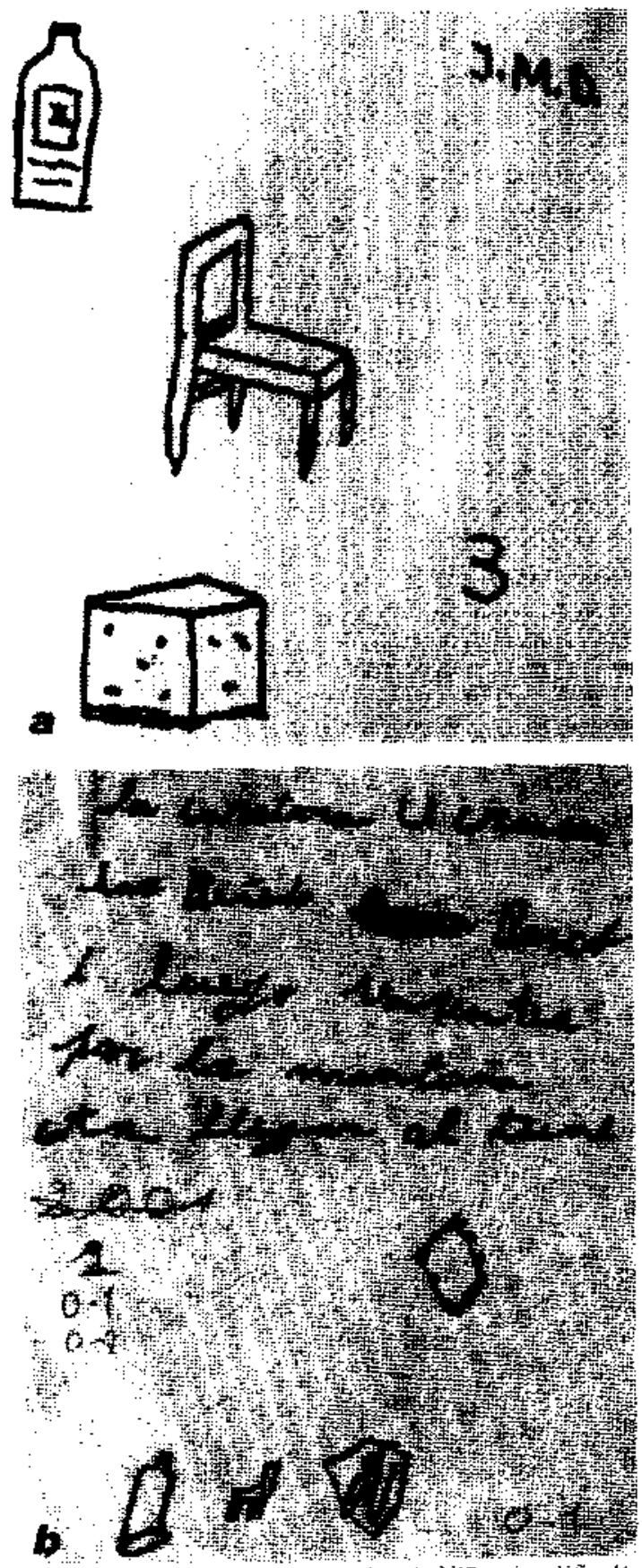

Figura 1. A) Dibujo tridumensional. Nino sin dificultad inicial en aprendizaje de lecto-escritura. B) Dibujo tridimensional. Nirio con difícultad injcial en aprendizaje de lecto-escritura. 


\section{COMENTARIO}

La comparación entre el nivel de aprendizaje inicial y una serie de indicadores del rendimiento escolar apreciados 7 años más tarde ha resultado positiva, directa y consistente desde el punto de vista estadístico en esta muestra. En otros términos, la mayor o menor facilidad para aprender a leer y a escribir en los primeros tres años de escolaridad aparece como indicador del futuro rendimiento académico en el niño con desartollo intelectual dentro de lonormal.

Los datos recogidos en esta investigación señalan que la falla inicial en el aprendizaje de la lecto-escritura, de manera especial cuando sobrepasa cierto nivel representado en esencia por los niños que no eran capaces de leer prácticamente nada a nivel del $3^{\mathrm{er}}$ año básico, significa a menudo una alteración que de una u otra forma se prolonga en el tiempo. Si bien, la capacidad individual de superación de esta disfunción inicia] severa es bastante variable (la experiencia diaria y las cifras de este estudio asi lo atestigua), no deja de ser importante que apenas la mitad de los niños con alteraciones de la lecto-escritura inicial tuvieran un nivel óptimo de esta habilidad 7 años más tarde. Este sólo hecho bastaría para explicar en ellos la falla en obtener promociones adecua. das y rendimientos óptimos, en especial si se utiliza exclusivamente la metódica de enseñanza escolar convencional, en base a la capacidad que tiene cada niño de leer y escribir hábilmente. Es claro, no obstante, que se trata algo más que una disfunción específica o restringida en la lectoescritura dado que cada aspecto analizado, incluyendo las operaciones aritméticas básicas y el dibujo tridimensional, ha resultado más alterado en los niños con dificultad inicial del aprendizaje de la lecto-escritura y tanto más cuanto mayor ha sido este impedimento.

Es importante considerar que este estudio aunque longitudinal, se refiere obviamente sólo a aquellos niños que se pudieron reexaminar. Un número importante de la muestra inicial no pudo ser reintegrado al estudio, resultando sugerente que de los 60 niños con dificultades marcadas de aprendizaje de la lecto-escritura inicial sólo se pudo captar a 20 de ellos siete años más tarde. Dado que el mayor porcentaje de niños con dificultades escolares ulteriores caen precisamente dentro de esta categoría, es lícito suponer que una alta proporción de malos lectores a nivel del tercer año básico hayan abandonado ulterjormente sus estudios, mientras que otros están en situación tan deficitaria que se negaron rotundamente a participar en este estudio. Este aspecto es muy importante dilucidarlo más a fondo, por lo que tenemos en marcha un proyecto para estudiar especificamente el punto.

Varios estudios, incluyendo nuestra experiencia, han reflejado que la deficiençia de aprendizaje y las fallas de compottamiento se acompañan frecuentemente de más signos de disfunción que en los niños controles ${ }^{8 \cdot 9 \cdot 10-11-12}$. Estas fallas sugieren la participación de algún proceso disfuncional del sistema nervioso central, o al menos una variante del desarrollo neuropsicológico, tanto en los aspectos motores, de lenguaje, perceptivos, de conceptualización de atención y conducta. Este enfoque del problema estaria apuntando por consiguiente hacia una cierta "organicidad" del trastorno original, to que apoyaría en cierto modo las teorías que indican que la dislexia de desarrollo tendría una base neuroanatómica $^{15}$. El estudio de conjunto de esta casuistica señala que las disfunciones expresadas en un niño con un nivel intelectual convenciona. mente normal, incluyendo la presencia de numerosos signos de alteración neurológica fina, permiten plantear la hipótesis que la falla de apren. dizaje injcial puede configurar parte de un síndrome neuropsicológico de características deficitarias. Esta disfunción es aparentemente subliminal, dado que se descartaron en este estudio los pacientes con disfunción neurológica abierta, con trastornos neurosensoriales y los sujetos con clara perturbación emocional.

Queda abierta la posibilidad que la dificultad en el aprendizaje inicial de la lecto eseritura no sea discriminativa respecto al rendimiento académico en plazo más prolongado que el de este estudio. Pero dado que la probabilidad estadísti. ca de un mal rendimiento ha resultado elevada y que ningún niño con dificultades de aprendizaje importantes en los 3 primeros años de escolaridad llegó a ser un alumno destacado, por su alto rendimiento académico, en el plazo de siete años, es de temer que en este lapso se hayan estructu. rado actitudes que reflejen sentimientos de inferioridad y subestimación que impidan la adecuada confrontación de los sujetos afectados a un medio social altamente competitivo, lo que minimizaría la hipotética recuperación neuropsicológica ad integrum a más largo plazo.

Estamos conscientes que el análisis realizado usando como indicador la capacidad para aprender la lecto-escritura inicial es una simplificación metodológica realizada con el objetivo de recono. cer la significancia aislada de este parámetro. Los casos que difieren de la tendencia estadística encontrada hace suponer un juego mucho más intrincado de otras variables biológicas, psicológicas, sociales y probablemente pedagógicas. Esperamos que los variados aspectos que iremos analizando en otras secciones del estudio nos ayuden a formarnos un juicio más amplio y equilibrado 
sobre los múltiples factores que eventualmente intervienen en el rendimiento escolar a largo plazo.

\section{RESUMEN}

119 escolares de inteligencia normal, provenientes de estratos económicosociales medio bajo y bajo, domiciliados en el sector norte de San. tiago, fueron evaluados en 1975 para medir el aprendizaje de la lecto-escritura, mientras cur. saban el segundo semestre del tercer Año Básico. Todos han sido seguidos longitudinalmente en su desarrollo, crecimiento y en la progresión académica desde su ingreso a la educación primaria en 1973 hasta la fecha. Además, en el curso de 1982 se les aplicó una serje de pruebas objetivas, a fin de valorar en forma homogénea las habilidades alcanzadas en lecto-escritura, en la resolución de problemas de aritmética básica y en el dibujo tridimensional de objetos simples.

En este estudio se ha constatado una relación estadistica directa, analógica y significativa entre el grado de eficiencia de la lecto-escritura a nivel del tercer año primario y los parámetros elegidos para evaluar el progreso académico ulterior. La mayor parte de los sujetos incluidos en este estudio que a la edad promedio de 8.9 años, no habjan aprendido a leer con facilidad exhibieron 7 años más tarde un rendimiento académico más bien limitado, ya que ninguno alcanzó los pun. tajes máximos en las pruebas efectuadas. Los menores rendimientos se apreciaron en la capa. cidad para resolver problemas de aritméticas sinples y dibujar objetos corrientes. Por el contrario, los nir̃os que aprendieron con facilidad obtuvieron resultados abiertamente superiores, aún cuando algunos tuvieron un rendimiento bajo, discrepancia que requiere de un análisis individualizado que está en desarrollo. Esta correlación pareciera ser independiente de la capacidad de lecto escritura alcanzada, ya que más de la mitad de los casos (53\%) con dificultades iniciales de aprendizaje podían leer $\mathrm{y}$ escribir aceptablemente 7 afios más tarde.

\section{AGRADECIMIENTO}

Los autores agradecen a la Srta. Rosa Llop, Programadora en el procesamiento computacional de'datos de este trabajo.

\section{REFERENCIAS}

1. Avendaño, A.; Valenzuela. C.; Figuerod, L. y Cols:: Estudio Longitudinal del crecimiento $y$ desarrollo de un $10 \%$ de los nifnos yue ingresan a la Enserianza Básica Fiscal. Area Hospitalaria Norte de Santiago. Pediatría (Santiago) 19:156, 1976.

2. Manterola, A.; Avendaño, A., Figueroa, L. y Cols.: Estudio sobre prevalencia y caracteristicas biológicas. Socioculturales y psicológicas de niños con insuficiente rendimiento escolar. Pediatría (Santiago) 19:83, 1976 .

3. Manterola, A.; Avendaño, A.: Valenzuela, C. y Cols: Estudio sobre prevalencia y caracteristicas biológicas, socioculturales y psicológicas de niños con insuficiente rendimiento escolar. I Parte: Diseño dc b lnvestigación. Rev. Chil. Pediatr. 52: 90,1981 .

4. Manterola, A.; Avendaño, A.; Valenzuela, C. $y$ Cols.: Estudio sobre prevalencia $y$ características biologrcas, socioculturales y psicológicas de niños con insuficiente rendimiento escolar. II Parte: Aspectos Socioculturales y Biológicos. Rev. Chil. Pediatr. 52:155, 1981.

S. Manterolo, A.; Avendaño, A.; Valenzuela, C. $y$ Cols.: Estudio sobre prevalencja y características biológicas, socioculturales y neuropsicológicas de nin̄os con insuficiente rendimiento escolar. IIla Parte: Características Neuropsicológicas. Rev, Chíl. Pediatr. 52: 250, 1981

6. Volenzuela, C. y Morales, I.: Estudio Bayesiano del rendimiento escolar según diez pruebas neurológicas. Rev. Chil. Pediatr. 52: 486, 1981.

7. Manterola. A.; Avendaño, A.: Íalenzuela, $C_{\text {. }} y$ Cols.: Verificación de relaciones entre Pruebas Neurológicas y Rendimiento Escolar. Rev. Chil. Psdiacr. 54: 20, 1983.

8. Rutter M., Graham P., Birch H.G.: Interrelations between chorriform syndrome, reading disabilities and psychiatric disorders in children of $8 \cdots 11$ years. Dev. Med. Child. Neurol. 8: 149, 1966.

9. Wolff P.H, y Hurwitz I.: The Choriform Syndrome. Dev, Med. Child. Neurol. 4: 160, 1966.

10. Adams R.M.; Kocsis J.J.: Estes R.E.: Soft Neurological Signs in Learning-diabled children and control. An. J. Dis. Child. 128: 614, 1974.

11. Peters J.E.: Romine J.S.; Dykman R.A.: A Special Neurological Examination of Children with Learning Disabilities. Dev. Med. Child. Neural. 19: B21, 1975 .

12. Korhoner T. y Sillampao M.: MBD-like behavior and neuropsychological perfomances. Acta Paedopsychiatr. 42: 75, 1976.

13. Rasmusien D., Gillberg C.: Porceptual motor and attentional deficits in seven-year ol children: Neurological and Ncurodevelopmental aspects. Dev. Med. Child. Neurol. 25: 315, 1983.

14. Monterola, A.; Auendaño, A.; Valenzuelo, C. y Cols.: Significado de la presencia de alteraciones neurológicas menores en al rendimiento escolar a largo plazo. (En ublicación).

15. Galaburda A.M., Kemper T.L.: Cyloarchitectonic Abnormalities in Developmental Dislexia: A Case Study. Arn. Neurol. 6: 94, 1979. 